\title{
Acute Radiation Syndrome in an Irradiated Minipig Model for Patients with Radiation Exposure
}

\author{
Hyosun Jang', Joong-sun Kim ${ }^{1,2}$, Sehwan Shim ${ }^{1}$, Won-seok Jang', Sun-Joo Lee', Jae Kyung Myung' ${ }^{1}$ Seung-Sook Lee', \\ Sunhoo Park ${ }^{1, *}$ \\ ${ }^{1}$ Laboratory of Radiation Exposure and Therapeutics, Korea Institute of Radiological and Medical Sciences, Seoul, Korea; ${ }^{2}$ Research center, Dongnam Institute \\ of Radiological and Medical Science, Busan, Korea
}

\section{Original Research}

Received December 20, 2016

Revision April 13, 2017

Accepted July 5, 2017

Corresponding author: Sunhoo Park

Laboratory of Radiation Exposure and Therapeutics, Korea Institute of Radiological and Medical Sciences, 75 Nowon-ro, Nowon-gu, Seoul 01812, Korea

Tel: $+82-2-3399-5803$

Fax: +82-2-3399-5870

E-mail: sunhoo@kirams.re.kr

This is an Open-Access article distributed under the terms of the Creative Commons Attribution NonCommercial License (http://creativecommons.org/ licenses/by-nc/4.0) which permits unrestricted noncommercial use, distribution, and reproduction in any medium, provided the original work is properly cited.

Copyright $\odot$ 2017The Korean Association for Radiation Protection

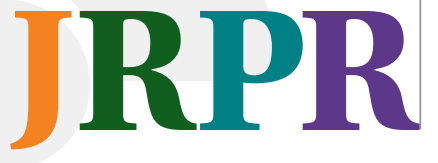

Background: Acute radiation syndrome (ARS) primarily refers to damage to the hematopoietic system, myeloid system, and gastrointestinal (GI) system caused by radiation exposure. Such damage progresses to become life-threatening. In particular, as the syndrome develops very rapidly - within several hours from radiation exposure-prompt and accurate diagnosis and treatment are needed, as is further research into appropriate diagnostic and treatment modalities.

Materials and Methods: Minipigs, which display human-like properties, underwent wholebody irradiation at 2 or $4 \mathrm{~Gy}$ (doses causing hematopoietic ARS) or at higher doses of 7 or 12 Gy. Changes in the blood cells and clinical symptoms were analyzed and we performed a necropsy when the animals succumbed to ARS.

Results and Discussion: The minipig irradiated with 2 Gy showed a decrease in white blood cells, including neutrophils, lymphocytes, and platelets in the early stages. However, the blood cell counts gradually increased and returned to normal values. The minipig irradiated with $4 \mathrm{~Gy}$ succumbed due to hematopoietic ARS. In contrast, the minipigs irradiated with 7 or 12 Gy exhibited clinical symptoms of combined GI damage and hematopoietic syndrome. Moreover, a characteristic pattern of platelet changes was observed in the 7 and 12 Gy irradiated minipigs.

Conclusion: The changes in the platelet count caused by radiation exposure observed in minipigs, which are hematologically and pathohistologically similar to humans, suggest that they can be used as a novel diagnostic criterion.

Keywords: Radiation, Minipig, Blood, Bone marrow

\section{Introduction}

With the growth of the nuclear power and radiation industry, as well as the increased risk of radiation accidents and nuclear terrorism, fear of radiation exposure is growing. Accordingly, it is important that victims of radiation exposure receive medical management by well-prepared personnel [1]. Radiation causes damage in various organs, depending on the dose of exposure and the part of the body that is irradiated. The damage that develops in the acute stage of an incident (within 2 weeks from the exposure) is referred to as acute radiation syndrome (ARS).

ARS affects the hematopoietic system, gastrointestinal (GI) system, skin, and neurovascular system [2]. In particular, hematopoietic ARS can have a significant impact on one's life, and may even occur with a relatively low dose of radiation exposure [3]. Hematopoietic cells, due to their constant division, are sensitive to radiation. After being 
exposed to radiation, the number of white blood cells (WBC), including neutrophils, decrease, which in turn increases the risk of infection. Additionally, the decrease in the platelet count leads to an increased bleeding tendency, which has been reported to be a major eventual cause of death.

Moreover, the GI effects of ARS can also be influence survival after radiation exposure [4]. Because the crypt cells of the GI system are sensitive to radiation [5], irradiation causes apoptosis of crypt cells, which in turn leads to villous atrophy, malabsorption, damage to the bowel wall by the loss of epithelial cells in the intestine, and inflammation. This ultimately results in exudative diarrhea.

Currently, granulocyte-colony stimulating factor (G-CSF) is used to treat damage of the hematopoietic system from radiation exposure, and it has been reported to be effective in the treatment of humans [6]. However, for GI damage by radiation, only symptomatic treatment-instead of treatment of the fundamental causes-is provided. According to the severity of the symptoms, various medications must be administered. Therefore, it is necessary to establish animal models for human of radiation exposure.

The commonly used animal models for radiation studies include rodents, such as mice or rats. However, as they are very different from humans anatomically and develop different patterns of damage in the bone marrow and hematopoietic system by irradiation, the use of these animals in research has significant limitations [7, 8]. Medium-sized animal models that simulate humans, such as apes and canines, have also been used for radiation research. Nonetheless, due to ethical issues and high management costs, such animals are also used to a limited extent in research.

Minipigs, since they are the most similar to humans both anatomically and physiologically [9], are used in place of humans in diverse areas of medical research, including insulin production and organ transplants. Moreover, minipigs can be used for studying clinical procedures; in particular, as their tissues can be sequentially collected over time, it is easy to observe associations between clinicopathological changes and radiation exposure. Minipigs have been maintained with limited genetic and microbiological characteristics and are widely used for research. Overall, minipigs are a popular mediumsized animal model that can replace ape or canine models.

Furthermore, previous studies have reported that the response of minipigs to radiation exposure is very similar to that of humans $[8,10,11]$. However, despite such similarities, research into radiation exposure using minipigs has mostly been limited to acute damage in the myeloid hematopoietic system at doses of $2 \mathrm{~Gy}$ or below [12]. Therefore, further studies are necessary to examine the effects of exposure to higher doses of radiation and the appropriate diagnosis and treatment for such irradiation.

Therefore, in this study, the authors observed hematological and pathophysiological changes in minipigs irradiated with 2 or $4 \mathrm{~Gy}$-doses at which acute myeloid hematopoietic syndrome occurs-and at higher doses of 7 or $12 \mathrm{~Gy}$.

\section{Materials and Methods}

\section{Experimental animals and irradiation}

Six-month-old male minipigs (PWG Genetic Korea, Seoul, South Korea) were used. The minipigs were housed in the specific-pathogen-free room of the animal facilities of the Korea Institute of Radiological Medical Sciences (KIRAMS) with a 12-hour light/12-hour dark cycle at a constant temperature $\left(22 \pm 1^{\circ} \mathrm{C}\right)$ and humidity $(50 \% \pm 10 \%)$, and were fed with sterilized feed and water purified with a 3-stage filter. This animal experiment was approved by the Animal Experiment Committee of the KIRAMS. The procedures were performed in accordance with the regulations of the Institutional Animal Care and Use Committee of the KIRAMS.

To secure the minipigs during irradiation, $2.5 \mathrm{mg} \cdot \mathrm{kg}^{-1}$ of Zoletil 50 (Virbak Korea, Seoul, South Korea) was injected intramuscularly for general anesthesia. To estimate the dose of radiation exposure accurately, a thermoluminescence dosimeter (TLD) (on 4 areas, including the chest, gluteus, back, and abdomen) and EBT film (12 units around the abdomen) were attached to the minipigs and fixed using frames. For irradiation, we used the method of Shim et al. method [10] as a reference: briefly, minipigs underwent bilateral whole-body gamma irradiation dose levels of 2, 4, 7, or $12 \mathrm{~Gy}$ at the rate of $130.61 \mathrm{cGy} / \mathrm{min}$, using a ${ }^{60} \mathrm{Co}$ gamma irradiator (Theratron 780, AECL, Ontario, Canada) (Figure 1).

At an irradiation of $2 \mathrm{~Gy}$, the TLDs on the chest, gluteus, back, and abdomen indicated 2.3, 2.5, 2.4, and 2.4 Gy, respectively. At $4 \mathrm{~Gy}$, the levels on the TLD were 4.4, 5.3, 5.5, and $5.5 \mathrm{~Gy}$, respectively. At $7 \mathrm{~Gy}$, the levels on the TLD were 8.7, 8.6, 10.0, and 9.5 Gy, respectively. At $12 \mathrm{~Gy}$, the levels on the TLD were 13.5, 14.3, 15.0, and $14.9 \mathrm{~Gy}$, respectively. These differences are consistent with the reported $20 \%$ error between the dose administered to the core to the dose registered on the skin surface $[13,14]$. 

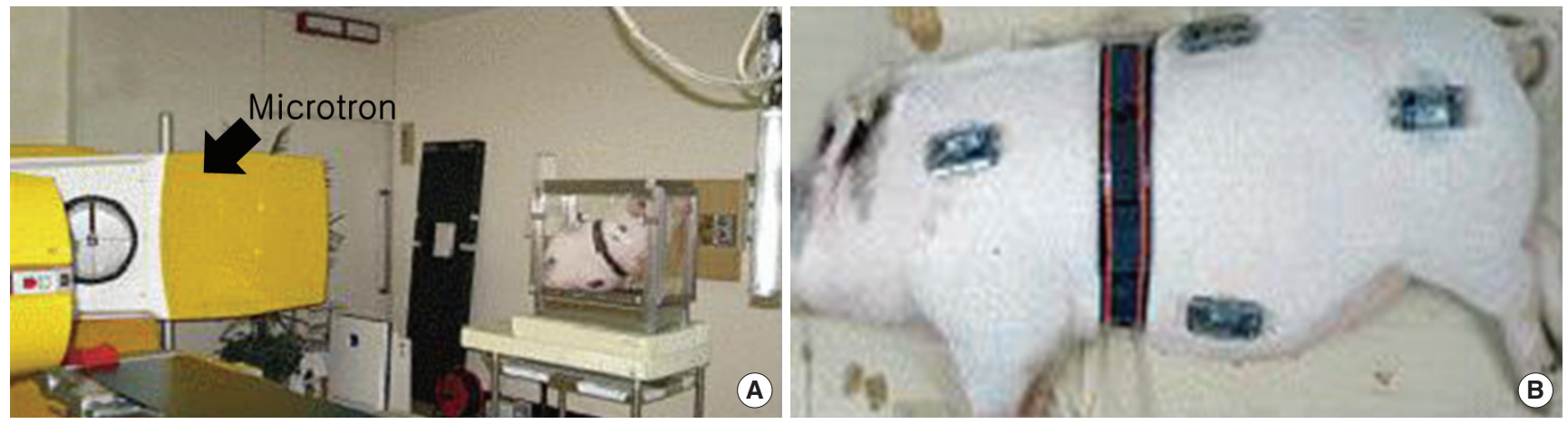

Fig. 1. Picture of (A) whole-body irradiation by microtron and (B) application of thermoluminescence dosimeter (TLD) on the chest, gluteus, back, and abdomen in minipig.

\section{Variables observed and examined}

Over the course of the experiment, we measured feed intake, clinical symptoms, and changes in the blood count before and after irradiation once a day. Before irradiation, we measured the peripheral blood cell count of the minipigs and used those values as a control to compare the counts after irradiation. Blood was collected at 30 minutes, 14 hours, and 24 hours after irradiation and once a day thereafter in $0.5-\mathrm{mL}$ capillary tubes ( $\mathrm{K}_{2}$ EDTA tube; BD Microtainer, Bridgeport, $\mathrm{NJ}$ ) that contained anticoagulant agents. The content was mixed carefully for 10 minutes. Then, red blood cells (RBC), WBC, neutrophils, lymphocytes, and platelets were counted using an automatic veterinary hematology analyzer, HEMAVET $^{\circledR}$ System (Drew Scientific Inc., Warrington, UK).

To observe histological changes in the bone marrow, we performed a bone marrow biopsy from the bilateral femurs at 30 minutes, 17 hours, 3 days, and 14 days after irradiation. When animals died, we performed a bone marrow biopsy immediately after death. We fixed the bone marrow tissues with formalin and sliced them. Then, we performed hematoxylin and eosin (H\&E) staining and immunostaining with Ki-67 to identify the proliferation of bone marrow cells.

\section{Results}

\section{Clinical and pathological changes}

In this study, we assessed the cause of death through observations of clinical symptoms and by performing necropsy of minipigs that underwent whole-body irradiation at different doses. The minipig irradiated with $2 \mathrm{~Gy}$ did not exhibit specific clinical symptoms. The minipig irradiated with $4 \mathrm{~Gy}$ did not show macroscopic changes in the intestinal mucosa on an endoscopic examination. Histologically, shortening of

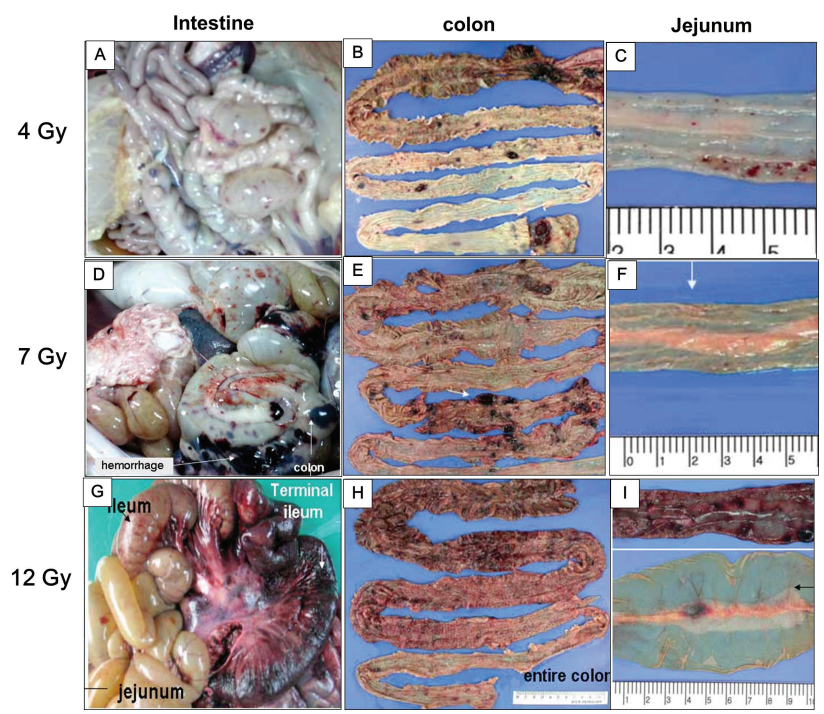

Fig. 2. Pathological alterations of the gastrointestinal system in minipigs irradiated with 4 Gy (A-C), 7 Gy (D-F), and 12 Gy (G-I) at necropsy.

the villi and reduction in the proliferation of crypt cells were observed; still, the overall absorption function remained intact. However, the animal succumbed at day 13 after irradiation. On the necropsy conducted to identify the cause of death, a bleeding tendency from each organ was observed (Figure 2A-C).

The minipig irradiated with 7 Gy developed fever, diarrhea, and hematuria at day 10. Furthermore, it succumbed at day 10 from irradiation. On necropsy, the bleeding tendency in each organ was remarkable (Figure 2D-F) and the minipig was found to have developed sepsis. The minipig irradiated with 12 Gy showed salivary secretions, loss of appetite, and vomiting in the early stages. Starting on day 5 , the animal developed fever and severe diarrhea. The animal succumbed on day 8 after irradiation and was found to have developed 
sepsis. Moreover, on necropsy, we observed that the minipig exposed at $12 \mathrm{~Gy}$ had severe bleeding in each organ, as well as loss and perforation of the intestinal mucosa (Figure 2G-I).

\section{Hematological changes}

To observe the hematological changes in the minipig models after high-dose, whole-body irradiation at $2 \mathrm{~Gy}$ (a sublethal dose for humans), $4 \mathrm{~Gy}$ (the median lethal dose), or higher doses of 7 or 12 Gy to each minipig, we measured changes in WBC including neutrophils and lymphocytes, platelets, and $\mathrm{RBC}$ over time according to dosage.

The WBC count gradually decreased to $1,280 \mu \mathrm{L}^{-1}$ on day 5 after irradiation in the minipig irradiated with $2 \mathrm{~Gy}$. The count remained at a nadir of $1,000 \mu \mathrm{L}^{-1}$, or slightly above, and gradually recovered after day 21 (Figure $3 \mathrm{~A}$ ). In the minipigs irradiated with the higher doses of 7 or $12 \mathrm{~Gy}$, the WBC count drastically dropped 24 hours after irradiation. On day 3, the count hiked momentarily, but decreased again, and the animals succumbed. In the minipig irradiated with $4 \mathrm{~Gy}$ (the median lethal dose), the WBC count decreased more slowly than in the minipigs irradiated with 7 or 12 Gy: on day 6 , the count dropped below $1000 \mu \mathrm{L}^{-1}$ and never recovered until the animal succumbed (Figure 3B).

Turning to the neutrophil count, the minipigs irradiated with 2 or 4 Gy showed similar patterns to those observed in humans: there was a momentary increase followed by a constant drop [15] (Figure 3C and D). The minipig irradiated with 2 Gy then maintained a nadir between day 5 and day 21. After day 21, the neutrophil count gradually increased, returning to normal values on day 70 and thereafter (Figure 3C). In contrast, in the minipig irradiated with $4 \mathrm{~Gy}$, the neutrophil count hiked temporarily and dropped drastically, never recovering until the animal succumbed on day 13 (Figure 3D). The minipigs irradiated with 7 or 12 Gy showed a constant decrease in the neutrophil count after irradiation. On day 5 and thereafter, the level remained at $1000 \mu \mathrm{L}^{-1}$ and below, and the animals succumbed on day 10 and day 8 , respectively (Figure 3D).

The time point when the neutrophil count dropped below $500 \mu \mathrm{L}^{-1}$ - the criterion for the use of G-CSF, the main treatment agent for ARS [16] —-was day 4 after irradiation for a dose of $12 \mathrm{~Gy}\left(100 \mu \mathrm{L}^{-1}\right)$ and day 5 after irradiation for the doses of 7 and $4 \mathrm{~Gy}\left(120 \mu \mathrm{L}^{-1}\right.$ and $360 \mu \mathrm{L}^{-1}$, respectively). These changes in the neutrophil count in the minipigs are similar to those that have been reported in humans. Moreover, the temporary increase of neutrophils in humans in the first 2 to 3 days after radiation exposure [3, 15] was also observed in the minipig model.

Lymphocytes are highly sensitive to radiation, and in all minipigs, on day 1 after irradiation, the lymphocyte count decreased to very low levels ( $2 \mathrm{~Gy}, 1,060 \mu \mathrm{L}^{-1} ; 4 \mathrm{~Gy}, 370 \mu \mathrm{L}^{-1} ; 7$ Gy, $360 \mu \mathrm{L}^{-1}$; and $\left.12 \mathrm{~Gy}, 190 \mu \mathrm{L}^{-1}\right)$. After day 1 , in the minipig irradiated with $2 \mathrm{~Gy}$, the lymphocyte count remained at 1,000 $\mu \mathrm{L}^{-1}$ and gradually increased starting on day 21 (Figure 3E). In the minipig irradiated with $4 \mathrm{~Gy}$, the count temporarily increased from day 1 to 4 , to reach $500-1,000 \mu \mathrm{L}^{-1}$ and then constantly dropped until the animal succumbed (Figure 3F). In the minipigs irradiated with 7 or $9 \mathrm{~Gy}$, the lymphocyte count temporarily increased on day 3 and remained at a nadir until the animals succumbed on day 10 and day 8 , respectively (Figure 3F).

Regarding changes in the platelet count, in the minipig irradiated with $2 \mathrm{~Gy}$, the level constantly dropped after the radiation exposure until day 14 , and remained at a nadir until day 30 . At day 30 and thereafter, the platelet count gradually increased and reached close to normal values at day 70 , which were then maintained (Figure 3G). Furthermore, there was a clear difference in the changes in platelets between $4 \mathrm{~Gy}$ (the median lethal dose) and the higher doses of 7 or 12 Gy. The minipig irradiated with 4 Gy showed a gradual and constant decrease in platelets. In contrast, the minipigs irradiated with 7 or 12 Gy showed a drastic drop in platelets after irradiation until day 2 , a temporary increase until day 5 , and a drastic decrease again until both animals succumbed (Figure 3H).

$\mathrm{RBC}$ are highly resistant to radiation, and the minipig irradiated with 2 Gy maintained normal $\mathrm{RBC}$ values even after irradiation (Figure 3I). Similarly, the minipig irradiated with 4 Gy maintained a normal RBC count until it succumbed. In contrast, in the minipigs exposed to the extremely high doses of 7 or $12 \mathrm{~Gy}$, the RBC count dropped drastically on day 1 after irradiation and gradually increased to reach normal values immediately before the animals succumbed (Figure 3J).

\section{Histological changes}

Biopsy of bone marrow tissue may cause problems such as infection and bleeding in patients exposed to radiation. Therefore, it is challenging to observe changes in bone marrow over time. In this study, we observed histological changes in bone marrow through bone marrow biopsies at different time points, based on the changes in peripheral blood in minipigs that underwent whole-body irradiation, in order to 

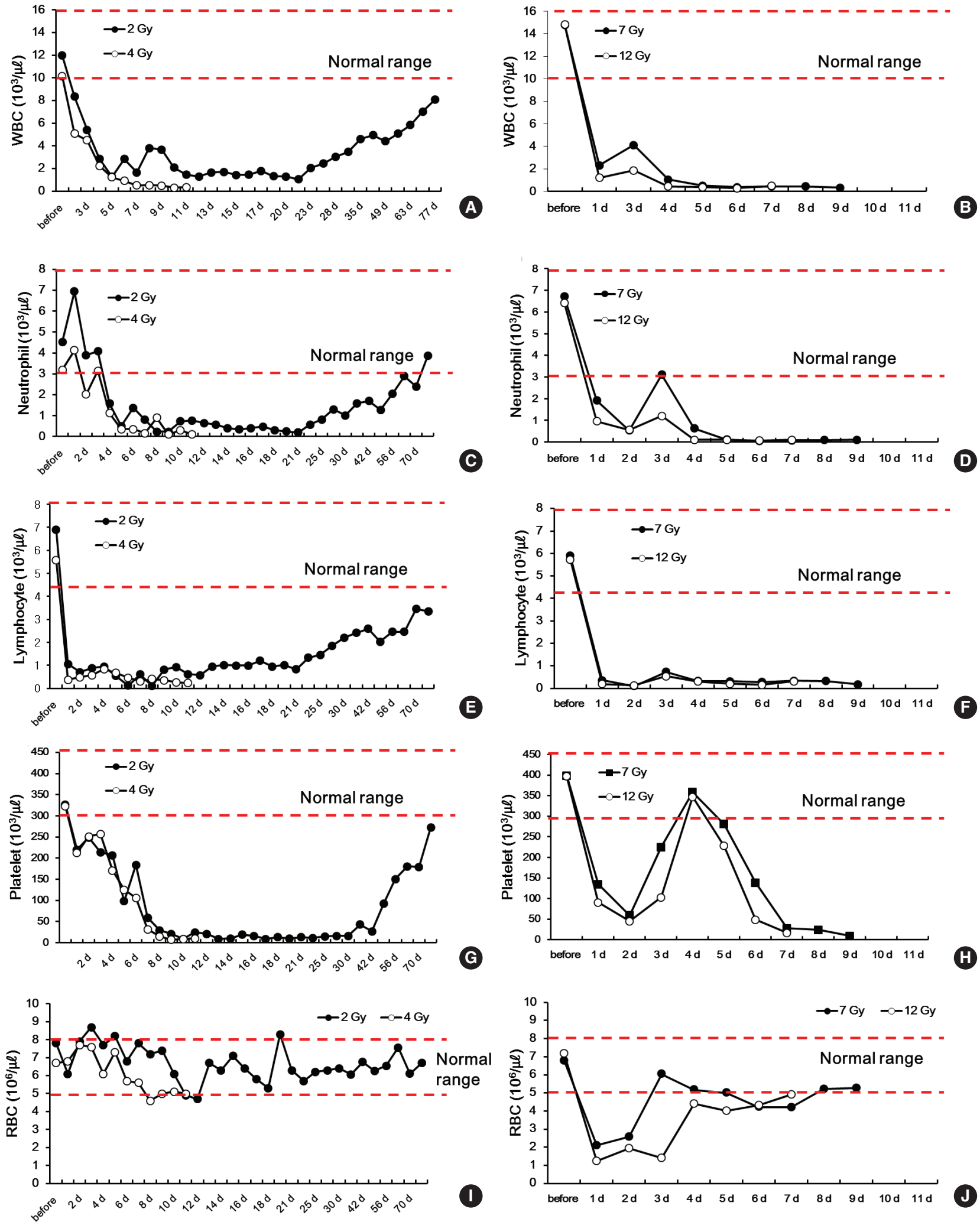

Fig. 3. Changes in white blood cells (WBC) (A, B), neutrophils (C, D), lymphocytes (E, F), platelets $(G, H)$ and red blood cells (RBC), and (I, J) in minipigs irradiated with 2, 4, 7, and 12 Gy. 


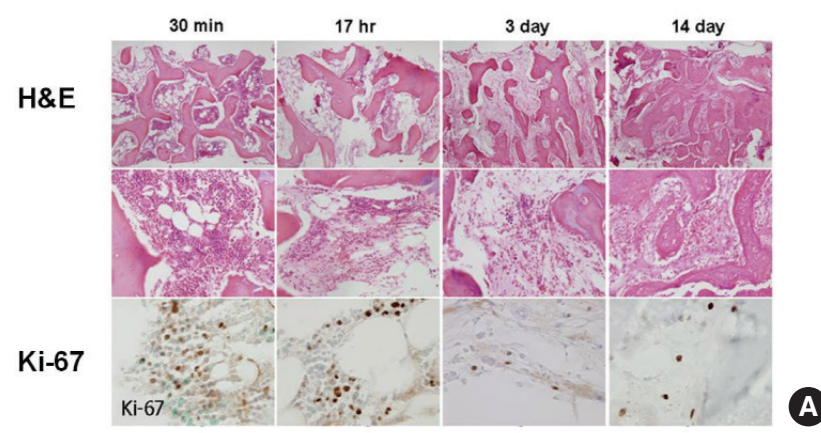

H\&E

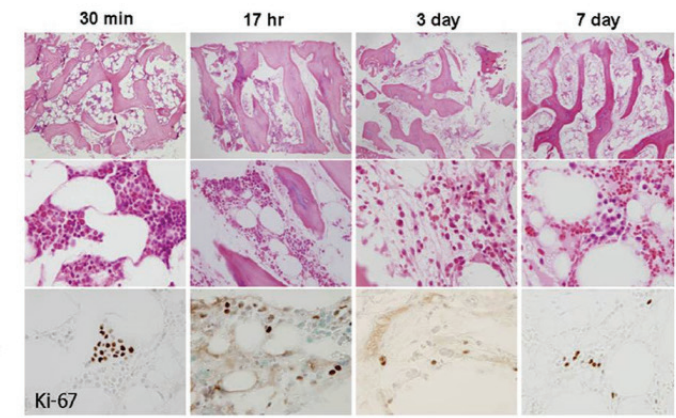

B

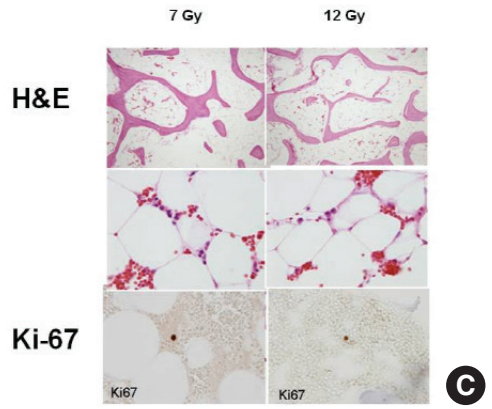

Fig. 4. Hematoxylin and eosin (H\&E) staining and Ki-67 immunohistochemistry in the bone marrow of the 2 Gy-irradiated minipig $(A)$, the 4 Gy-irradiated minipig (B), and the 7 and 12 Gy-irradiated minipigs (C).

directly compare the changes observed in the complete blood counts and the conditions of the bone marrow.

After irradiation with 2, 4, 7, or $12 \mathrm{~Gy}$, changes in the bone marrow cells over time were examined through H\&E staining and Ki-67 staining, a proliferation marker. In minipigs irradiated with 2 or $4 \mathrm{~Gy}$, the myeloid hematopoietic cells disappeared drastically, starting at 30 minutes after irradiation; at the 17-hour point, only a minimal number of myeloid cells remained. On day 3 after irradiation, only some plasmacytoid cells and erythroblasts were observed; Ki-67 positive cells were hardly found (Figure 4A and B). For minipigs irradiated with 7 or $12 \mathrm{~Gy}$, bone marrow tissues were obtained when they succumbed. In the histological analysis, we found that the bone marrow of these 2 minipigs was replaced with adipose cells, with a very small number of plasmacytoid myeloid cells. Moreover, few Ki-67 positive cells were found in the bone marrow (Figure 4C).

\section{Discussion and Conclusion}

In this study, we observed hematological and histopathological changes in response to radiation exposure in minipigs according to the dose in order to establish minipigs as a human-like model for ARS. We performed whole-body irradiation of minipigs with $2,4,7$, or $12 \mathrm{~Gy}$, and observed the manifestations of clinical symptoms and hematological changes.

We chose 2 and $4 \mathrm{~Gy}$ as radiation doses corresponding to the development of acute myeloid hematopoietic system syndrome, as well as higher doses of 7 and $12 \mathrm{~Gy}$. In this model, we compared changes in blood components (WBC, RBC, neutrophils, lymphocytes, and platelets) and the histopathology of the internal organs. The WBC count of minipigs decreased drastically after exposure to radiation, similar to the changes in humans [18].

Neutrophils temporarily increased in the early stages of radiation exposure in the minipigs irradiated with 2 or $4 \mathrm{~Gy}$. This is the pooling phenomenon, in which blood cells stored in the spleen leak into the peripheral vessels [19], and this phenomenon also occurs in humans. The minipig irradiated with 2 Gy later recovered. The minipig irradiated with $4 \mathrm{~Gy}$ succumbed when its neutrophil count dropped to a nadir. On the contrary, in minipigs exposed to higher doses of 7 or $12 \mathrm{~Gy}$, there was a delay in the initial increase of neutrophils, which occurred on day 3 after irradiation. The changes in lymphocytes, the component most sensitive to radiation, exhibited the most similar response to human data.

Within the initial 24 hours after irradiation, the lymphocyte count drastically dropped in all animals exposed to radiation. In the minipig irradiated with $2 \mathrm{~Gy}$, the count slowly recovered. In contrast, in the minipigs exposed to higher doses of 4,7 , or $12 \mathrm{~Gy}$, the count constantly remained at low levels until the minipigs succumbed. The changes in platelets exhibited clear differences by dose. In the minipig irradiated with $2 \mathrm{~Gy}$, although the recovery was slow, the count returned to normal values after 70 days. In the minipig irradiated with $4 \mathrm{~Gy}$, the low level continued. This is very similar to the pattern that appears in humans exposed to radiation.

However, in the minipigs exposed to 7 or $12 \mathrm{~Gy}$, in which ARS developed, the platelet count temporarily increased to 
normal values on day 4 after irradiation, followed by a sudden drop, and remained at low levels. The RBC of humans and minipigs are known to be most resistant to radiation among all hematocytes $[12,20]$. Similarly, in this study, the RBC levels of the minipigs exposed to 2 or 4 Gy remained within the normal range. However, in minipigs irradiated with 7 or $12 \mathrm{~Gy}$, there was a temporary drop in the RBC count in the initial stage, after which the count returned to a normal level. With higher doses, the recovery took longer.

Based on these findings, we demonstrated that hematological changes in minipigs in response to radiation exposure are very similar to those of humans. In particular, the changes in lymphocytes were almost identical to those observed in humans. We expect that this pattern of changes in hematocytes according to the radiation exposure dose in minipigs may serve as a parameter for estimating exposed doses. Currently, due to the lack of patients exposed to radiation, it is impossible to compare and review the results of various types of treatment. Therefore, based on the results of this study, we suggest that minipig models may be a proper animal model for human ARS.

The results of the histological analysis of the bone marrow showed that the myeloid hematopoietic cells dramatically disappeared 30 minutes immediately after irradiation; only less than $1 \%$ of the initial total remained at the 17 -hour point. On day 3, only parts of plasmacytoid cells and erythroblasts were observed, and Ki-67 positive cells were hardly found. In comparison to the results of the WBC count, bone marrow damage was found to occur much earlier than blood cell damage in peripheral vessels.

Furthermore, minipigs irradiated with 4 Gy succumbed to sepsis, without clinical symptoms such as vomiting or diarrhea. This is consistent with the report that in humans exposed to doses lower than 6 Gy, GI symptoms do not occur, and only acute hematopoietic symptoms develop [21]. In contrast, the minipigs exposed to 7 or 12 Gy showed clinical symptoms of vomiting and diarrhea caused by GI tract damage. Additionally, general atrophy of the intestinal mucosa, enterobacterial infection, and sepsis were observed. This suggests that for radiation exposure, it is important not only to treat the hematopoietic system, but also to focus on recovery and protection of GI tract, with the goal of both treating GI damage and preventing infections.

This study suggests that the hematological and pathohistological changes in response to radiation exposure in minipigs are very similar to those of humans. Based on these re- sults, we propose that minipigs can be used as a human-like animal model for studies of the mechanisms of damage due to radiation exposure, as well as diagnosis and treatment.

\section{Acknowledgements}

This study was supported by grant from the Korea Institute of Radiological and Medical Sciences (KIRAMS), funded by Ministry of Science, ICT, and Future Planning, Republic of Korea (1711045573;1711045551/50535-2017).

\section{References}

1. Dörr HD, Meineke V. Appropriate radiation accident medical management: Necessity of extensive preparatory planning. Radiat. Environ. Biophys. 2006;45(4):237-244.

2. Dörr H, Meineke V. Acute radiation syndrome caused by accidental radiation exposure - therapeutic principles. BMC Medicine. 2011;9:126.

3. Dainiak N. Hematologic consequences of exposure to ionizing radiation. Exp. Hematol. 2002;30(6):513-528.

4. Mettler FA Jr, Voelz GL. Major radiation exposure-What to expect and how to respond. N. Engl. J. Med. 2002;346(20):15541561.

5. Potten CS. Structure, function and proliferation organization of mammalian gut. In: Radiation and gut. 1st Ed. Amstredam, Netherland. Elsevier Science. 1995;1-31.

6. Reeves G. Overview of use of G-CSF and GM-CSF in the treatment of acute radiation injury. Health. Phys. 2014;106(6):699703.

7. Vigneulle RM, Herrera J, Gage T, MacVittie TJ, Taylor P, Zeman G, Nold JB, Dubois A. Nonuniform irradiation of the canine intestine. Radiat. Res. 1990;121(1):46-53.

8. Williams JP, et al. Animal models for medical countermeasures to radiation exposure. Radiat Res. 2010;173(4):557-578.

9. Swindle MM, Makin A, Herron AJ, Clubb FJ Jr, Frazier KS. Swine as models in biomedical research and toxicology testing. Vet. Pathol. 2012;49(2):344-356.

10. Shim S, Jang WS, Lee SJ, Jin S, Kim J, Lee SS, Bang HY, Jeon BS, Park S. Development of a new minipig model to study radiationinduced gastrointestinal syndrome and its application in clinical research. Radiat. Res. 2014;181(4):387-395.

11. Jones SR, George RE, West JE, Verrelli DM. The relative effectiveness of fission neutrons for gastrointestinal death in miniature pigs. Radiat. Res. 1972;50(3):504-518.

12. Moroni M, Lombardini E, Salber R, Kazemzedeh M, Nagy V, Olsen C, Whitnall MH. Hematological changes as prognostic indicators of survival: similarities between Gottingen minipigs, humans, and other large animal models. PLoS One. 2011;6(9): 
e2521.

13. Kry SF, Smith SA, Weathers R, Stovall M. Skin dose during radiotherapy: A summary and general estimation technique. J. Appl. Clin. Med. Phys. 2012;13(3):20-34.

14. Court LE, Tishler R, Xiang H, Allen AM, Makrigiorgos M, Chin L. Experimental evaluation of the accuracy of skin dose calculation for a commercial treatment planning system. J. Appl. Clin. Med. Phys. 2008;9(1):2792.

15. Goldman M. Ionizing radiation and its risks. West. J. Med. 1982; 137(6):540-547.

16. Dainiak N, et al. First global consensus for evidence-based management of the hematopoietic syndrome resulting from exposure to ionizing radiation. Disaster. Med. Public. Health. Prep. 2011;5(3):202-212.

17. Dörr H, Lamkowski A, Graessle DH, Bennett A, Shapiro A, Farese AM, Garofalo M, MacVittie TJ, Meineke V. Linking the hu- man response to unplanned radiation and treatment to the nonhuman primate response to controlled radiation and treatment. Health. Phys. 2014;106:129-134.

18. Vorobiev AI. Acute radiation disease and biological dosimetry in 1993. Stem Cells. 1997;15(2):269-274.

19. Tubiana M, Lalanne CM. Hematological evolution of patients exposed to whole-body irradiation for organ transplantation. Ann. Radiol. 1963;6:561-565.

20. Carsten AL. Acute lethality-The hematopoietic syndrome in different species. In: response of different species to total body irradiation. 1st Ed. Groningen, Netherland. Kluwer Academic. 1984;61.

21. Donnelly EH, Nemhauser JB, Smith JM, Kazzi ZN, Farfán EB, Chang AS, Naeem SF. Acute radiation syndrome: assessment and management. South Med. J. 2010;103(6):541-546. 